\title{
Palliative Care Assessment Tools for Older Adults:
}

\section{ATTR A Review}

Dakessian Sailian S. RN, MPH; Abu-Saad Huijer H. RN, PhD, FEANS, FAAN;

Dhaini S. RN, PhD; Adra M. RN, PhD

American University of Beirut- Hariri School of Nursing

\section{Background}

-The global population of people aged 60 years and older is expected to more than double by year 2050 .

-Prevalence of multiple chronic diseases is substantial among older adults leading to dependence and decreased quality of life. This implies that palliative care is central necessity to the provision of quality care in this population.

-Assessing older adults' palliative care needs is the first step to delivering comprehensive care.

\section{Purpose}

-The aim of the review is to identify and describe existing assessment tools used to measure palliative care needs of older adults, highlighting their scope, content, and psychometrics.

\section{Methods}

- Systematic review of English language articles published from January 2000 to 2016 was conducted by two separate authors.

- Databases searched were CINAHL, Medline by Ovid, Psychinfo and Academic Search Complete.

- All papers describing assessment tools for older adults in palliative care context, self-administered or by caregivers, irrespective of diagnosis, severity of the illness, or setting were included.

\section{Prisma Flowchart}

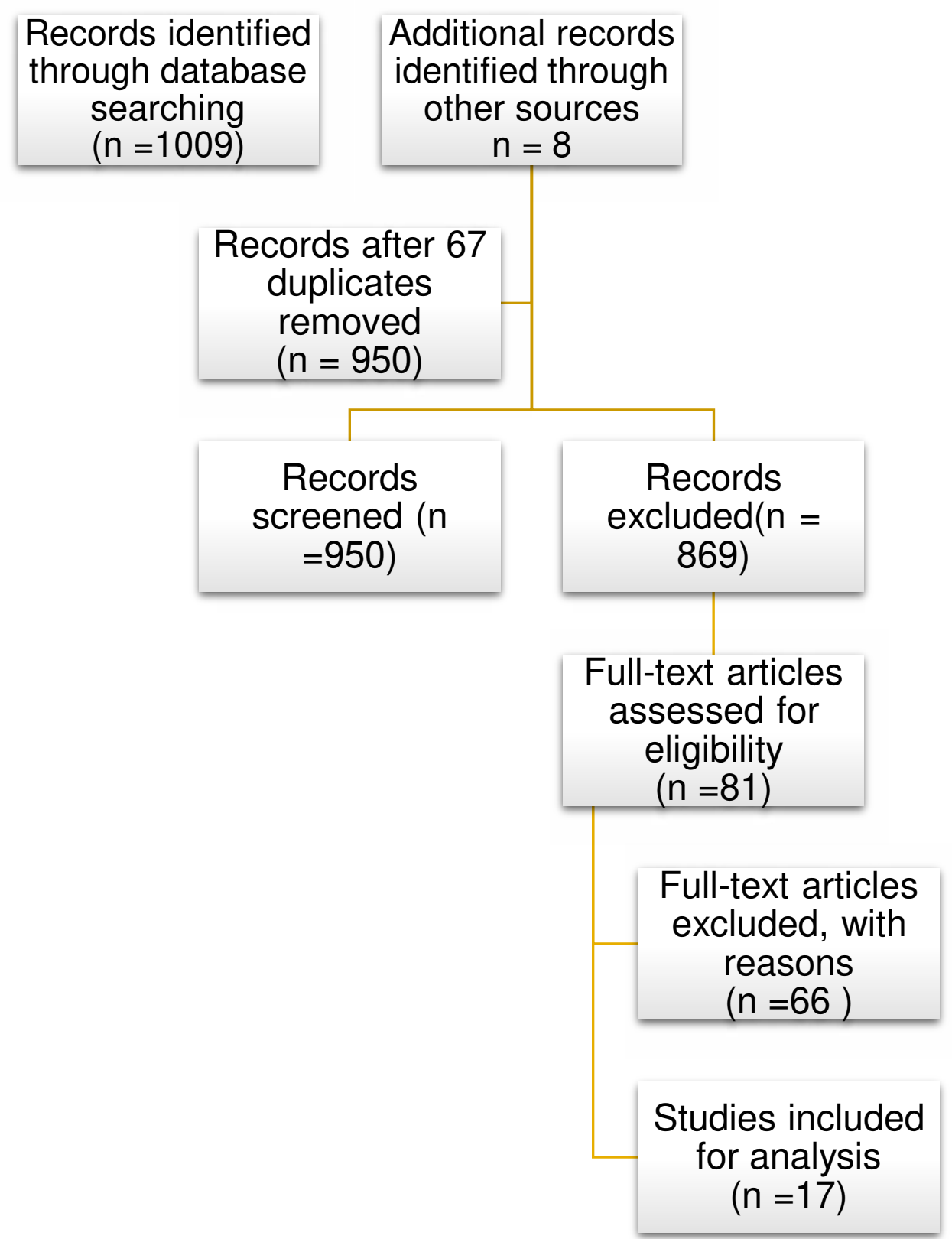

\section{Results}

- The initial search yielded 950 articles.

- 17 articles met the inclusion criteria, describing nine palliative care assessment tools used for older patients in various settings.

\begin{tabular}{|c|c|}
\hline POS & $\begin{array}{c}\text { - Palliative Care Outcome } \\
\text { Scale }\end{array}$ \\
\hline RAI-PC & $\begin{array}{c}\text { - Resident Assessment } \\
\text { Instrument for Palliative }\end{array}$ \\
\hline MQLS & $\begin{array}{c}\text { McMaster Quality of Life } \\
\text { Scale }\end{array}$ \\
\hline ASPE & $\begin{array}{c}\text { - Assessment Symptoms } \\
\text { Palliative Elderly }\end{array}$ \\
\hline NEST & $\begin{array}{c}\text { - Needs Near the End-of- } \\
\text { Life Screening Tool }\end{array}$ \\
\hline MQOL & $\begin{array}{c}\text { McGill Quality of Life } \\
\text { Index }\end{array}$ \\
\hline ESAS & $\begin{array}{c}\text { - Edmonton Symptom } \\
\text { Assessment Scale }\end{array}$ \\
\hline MQLC-E & $\begin{array}{c}\text { - Modified Quality-of-Life } \\
\text { Concerns in the End of } \\
\text { Life }\end{array}$ \\
\hline
\end{tabular}

\section{Conclusion}

- It can be concluded that no ideal tools are available to allow care providers to detect palliative care needs at an earlier stage in older adults.

\section{-The POS and RAl tools demonstrated better comprehensiveness and sensitivity to change in comparison to others.}

-In summary, this review
demonstrates that further
research is needed to create
robust tools for early detection of
palliative care needs. The
clinical relevance and cultural
sensitivity need to be studied
with regard to diverse countries
and cultures. Thus, instrument
validation is highly endorsed in
palliative care diagnosis or
disease trajectory.

\section{References}

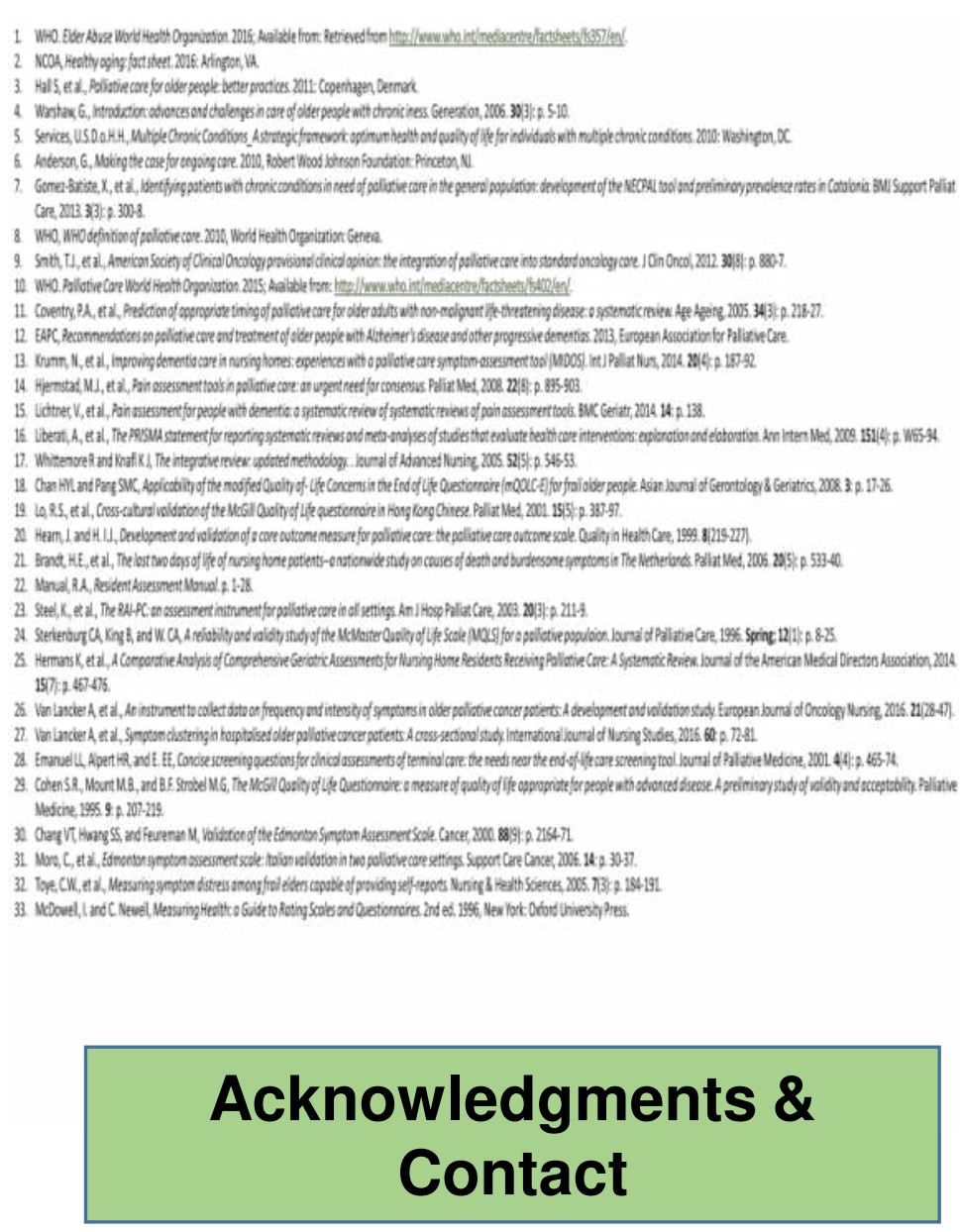

This manuscript did not receive any funds.

Poster is prepared by

Silva Dakessian Sailian RN, MPH Clinical Instructor

Hariri School of Nursing; American University of Beirut

Tel: +961-1-374374 extension 5967

Fax : +961-1-744476E-Mail:

silva.dakessian@aub.edu.lb 